\title{
Des lignées apocaryogamiques et leur ségrégation dans la population de l'Acrosipbonia arcta, Chlorophycée marine d'Héligoland
}

\author{
S. JónSSON \\ Faculté des Sciences, Laboratoire de Biologie Végétale Marine; Paris, France
}

\begin{abstract}
Apocaryogamic strains and their segregation in the population of the marine chlorophycean Acrosipbonia arcta from Helgoland. Nuclear studies of zygotes obtained from the wild type of Acrosiphonia arcta (DiLLw.) J. AG. from Helgoland were carried out in order to test the hypothesis that these algae might be of apocaryogamic origin. It is shown that caryogamy may be completely suppressed in zygotes of this species, although plasmogamy is normally carried out. The two pronuclei which do not fuse, become definitively separated after the first segmentation of zygote. In culture, these zygotes develop directly into filamentous plants which are morphologically similar to those from which they originate. These plants possess haploid nuclei with $\mathrm{n}=5$ chromosomes. The same chromosome number is found in the mother plants. Since successive generations continue to reproduce by apocaryogamy, it is suggested that genetically isolated micto-haploid strains occur in the $A$. arcta population studied. Origin and segregation of such strains are discussed.
\end{abstract}

\section{INTRODUCTION}

Des études antérieures (Jónsson 1959, 1962) ont permis de montrer que le cycle de base de l'Acrosiphonia spinescens (KüTZ.) KJELLM. de Roscoff (France) est digénétique et hétéromorphe. Ce cycle se caractérise par l'alternance de gamétophyte filamenteux (Acrosiphonia) et de sporophyte unicellulaire, ce dernier ayant été considéré autrefois comme une espèce distincte sous le nom de Codiolum petrocelidis, endophyte de l'algue rouge encroûtante, le Petrocelis cruenta. Il a aussi été établi que le gamétophyte est haploïde $(n=5)$ et monoïque et que les gamètes, de taille légèrement inégale, naissent dans des gamétocystes qui sont de sexes antagonistes. De plus, il a été démontré que la caryogamie s'accomplit normalement dans le zygote codioloïde et que la méiose a lieu dans le sporophyte diploïde, lors de la première division du noyau unique, au moment de la formation des spores quadriflagellées qui assurent le passage au gamétophyte (Jónsson 1970).

Ces études ont d'autre part montré que le cycle fondamental de l'A. spinescens peut être l'objet de complications portant sur la reproduction accessoire du gamétophyte. Cette reproduction se manifeste par la possibilité d'un développement direct de nouvelles générations filamenteuses qui sont morphologiquement similaires aux 
gamétophytes haploïdes. Les plantes ainsi nées peuvent être issues, chose imprévue, de faux zygotes où la plasmoganie a normalement lieu, mais non la caryogamie (Jónsson 1964). Cependant, elles ne paraissent pas constituer des lignées distinctes et stables car, en culture, elles sont capables de se comporter comme les gamétophytes normaux en produisant des sporophytes codioloïdes, effectuant ainsi un retour au cycle sexué normal (non publié). De plus, de telles lignées n'ont pas été trouvées dans la nature à Roscoff. Enfin, ces études ont montré que la reproduction apocaryogamique de $l^{\prime} A$. spinescens n'accuse pas un caractère obligatoire et qu'elle n'est jamais prépondérante dans le cycle sexué normal, et ceci indépendamment des conditions de culture.

Ces études ont été reprises et menées parallèlement à Héligoland par KornManN (1962, 1964, 1965) chez l'Acrosiphonia arcta (Dillw.) J. AG. Cette espèce ne diffère pas morphologiquement ni cytologiquement de l'A. spinescens dont elle est souvent considérée comme conspécifique et synonyme.

Les observations sur la reproduction de l'A. arcta ont tout d'abord permis de constater que les zygotes obtenus dans un lot de culture sont susceptibles de se développer directement en plantes filamenteuses sans passer par le stade codioloïde (Kornmann 1962). Ces résultats ont initialement été interprétés par cet auteur dans le cadre d'un cycle isomorphe, puis ultérieurement dans celui d'un cycle monogénétique et diplophasique («Diplont») (KornMann 1964). L'absence de stade codioloïde dans ce cycle a en même temps conduit KornManN à réfuter les résultats obtenus auparavant à Roscoff relatifs à l'existence d'un sporophyte codiolö̈de et d'un cycle digénétique et hétéromorphe chez l'A. spinescens. Selon cet auteur, la présence de générations codioloïdes, observée chez cette espèce, serait simplement imputable à des contaminations intervenues dans les cultures! Mais cette interprétation a dû être rapidement abandonnée. En effet, l'obtention inattendue par Kornmann (1965) d' $A$. arcta à partir de zoide issu d'un endophyte codiolö̈de du Petrocelis bennedyi d'Héligoland, a permis de démontrer la réalité d'un cycle de base digénétique et hétéromorphe chez ces algues et de confirmer ainsi les résultats déjà obtenus à Roscoff chez l'A. spinescens. Ce cycle, cependant, s'avère extrèmement rare à Héligoland, les gamétophytes filamenteux n'ayant pas été, selon cet auteur, trouvés dans la nature. Toutefois, il existerait, selon Kornmann, deux cycles différents au sein de l'A. arcta d'Héligoland, correspondant à deux formes différentes, voire deux espèces: $A$. arcta forme $\mathrm{A}$, à cycle monogénétique et diplophasique («Diplont») et $A$. arcta forme $\mathrm{B}$, à cycle digénétique et hétéromorphe, avec sporophytes codioloïdes.

Les investigations menées sur la reproduction de l'A. arcta par KorNMANN n'ont, cependant, pas été étayées d'observations caryologiques. C'est pourquoi il était permis de penser, par analogie avec ce qui avait été établi chez l'A. spinescens de Roscoff, que les plantes flamenteuses obtenues à plusieurs reprises à Héligoland par KorNMANN, à partir de zygotes à développement non codioloïde de l'A. arcta, puissent être de nature apocaryogamique, et que ce mode de reproduction ne s'inscrivît pas nécessairement dans le cadre d'un cycle prétendu monogénétique et diplophasique.

En vue de vérifier cette hypothèse, j'ai donc étudié la reproduction de l'A. arcta au moyen de cultures et d'observations nucléaires, en insistant tout particulièrement sur le problème de la caryogamie. Une note préliminaire a été publiée à ce sujet (JónsSON 1968). 
N.B. - Dans une publication récente (Kornmann 1970), parue après le dépôt du présent manuscrit, KoRNMANN abandonne l'idée d'un cycle monogénétique et diplophasique chez l'A. arcta. D'après cet auteur l'espèce se reproduirait selon un cycle monogénétique et haplophasique. Pour appuyer cette nouvelle interprétation KoRNMANN présente un montage photographique destiné à prouver la possibilité de caryogamie tardive dhez cette espèce. Cependant ce document ne permet pas de conclure à la fusion incontestable des noyaux gamétiques. En effet, les quelques figures considérées comme de vrais zygotes uninucléés (par ex. Fig. 2,6) pourraient être interprétées comme des faux zygotes binucléés vus de profil et dont les deux noyaux sont plus ou moins superposés. De plus, aucune figure ne montre des zygotes à noyau unique en division, et encore moins est-il possible de déceler une activité méiotique dans les noyaux. L'existence de quatre noyaux dans un zygote non cloisonné n'est pas une preuve de méiose, quand les zygotes sont initialement binucléés. Le comportement de ces zygotes diffère beaucoup de celui des vrais zygotes observés dans le cycle monogénétique et haplophasique de l'Acrosipbonia, et en particulier de l'A. sonderi d'Islande (Jónsson 1969, travail non cité par KorNmanN). Il faut aussi noter que les zygotes figurés par KORNMANN ne sont pas codioloïdes contrairement à tous les zygotes véritables observés jusqu’à maintenant dans le genre Acrosipbonia. Les très nombreux zygotes binucléés, d'âge différent, visibles sur les photographies présentées par Kornmann, plaident, par contre, en faveur de la défaillance caryogamique chez l'A. arcta, et confirment notre point de vue. Enfin il faut remarquer qu'un cycle monogénétique et haplophasique n'exclut pas l'existence de lignées apocaryogamiques parallèles. Le cas est déjà connu chez l'A. sonderi.

\section{RESULTATS}

\section{Obtention des gamètes et des zygotes}

Le matériel étudié a été récolté à Héligoland, puis envoyé à Paris, grâce à l'obligeance du Dr. Kornmann (Biologische Anstalt Helgoland). Ce matériel était fertile en arrivant à Paris, certains gamétocystes ayant même libéré leurs gamètes (Fig. 1). De plus, des éléments unicellulaires, arrondis, souvent avec deux stigmas visibles, et rappelant des zygotes, se trouvaient fixés sur les filaments de ces plantes.

Des rameaux portant chacun plusieurs gamétocystes ont été immédiatement isolés sur lame, en milieu de culture frais («Erdschreiber»), puis placés à la température de $+10^{\circ} \grave{a}+12^{\circ} \mathrm{C}$, et soumis à un éclairage de 1500 lux et à une photopériode de 14 heures d'éclairement journalier. Les gamétocystes ont émis au bout de quelques heures un grand nombre de gamètes biflagellés, chacun à un stigma net. Ceci a permis l'obtention de six lots unialgaux et d'un lot plurialgal. Cependant en aucun des cas observés il n'a été possible de déceler de copulations entre les gamètes. Ces derniers se sont simplement fixés sur le substrat, soit du côté de la lumière, soit du côté opposé à celle-ci.

Lors d'un second essai, des rameaux non fertiles de même thalle ont été placés dans des conditions analogues et en milieu nutritif fréquemment renouvelé. La crois- 
sance des algues a alors rapidement repris, accompagnée de divisions actives des cellules.

Une partie de ce matériel, fixée au mélange de WestBrook, puis colorée par la méthode nucléale classique de Feulgen, a montré des divisions somatiques. Comme chez tous les Acrosiphonia celles-ci sont précédées par la migration des noyaux au

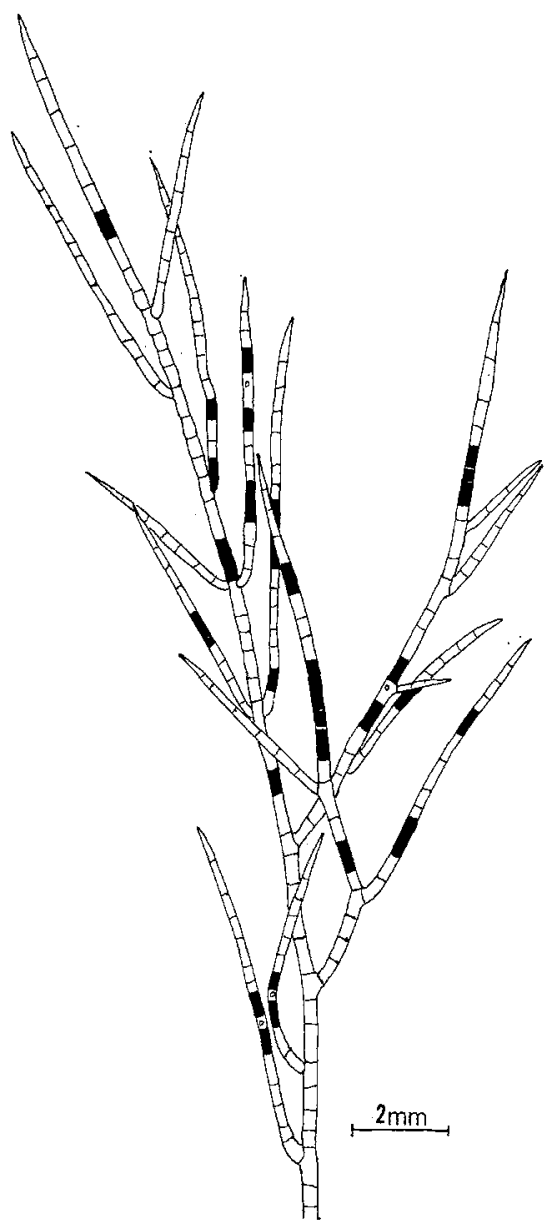

Fig. 1: Acrosiphonia arcta. Un aspect du thalle provenant de la nature; gamétocystes en noir. (Observation vitale)

centre de la cellule (Jónsson 1962). Au cours de ces divisions il a été possible de dénombrer 5 chromosomes par noyau (Fig. 2 A). En même temps il a été permis de constater que les éléments de nature apparemment zygotique trouvés sur les filaments de ces algues contenaient, chacun, nettement, deux noyaux au repos (Fig. 2 B).

L'autre partie du matériel, maintenue en culture, est devenue fertile au bout de cinq jours (Fig. 2 C). Toutefois les gamétocystes ne mûrissent pas tous en même temps. 
Quelques rameaux fertiles de ce matériel, fixés et colorés, ont permis de saisir quelques stades de la gamétogenèse. Celle-ci se caractérise par des divisions presque synchrones des noyaux. A un stade avancé de la gamétogenèse il a été possible de déterminer un nombre chromosomique égal à celui observé lors des divisions somatiques (Fig. 2 D). Ce nombre apparaît ainsi haploïde.

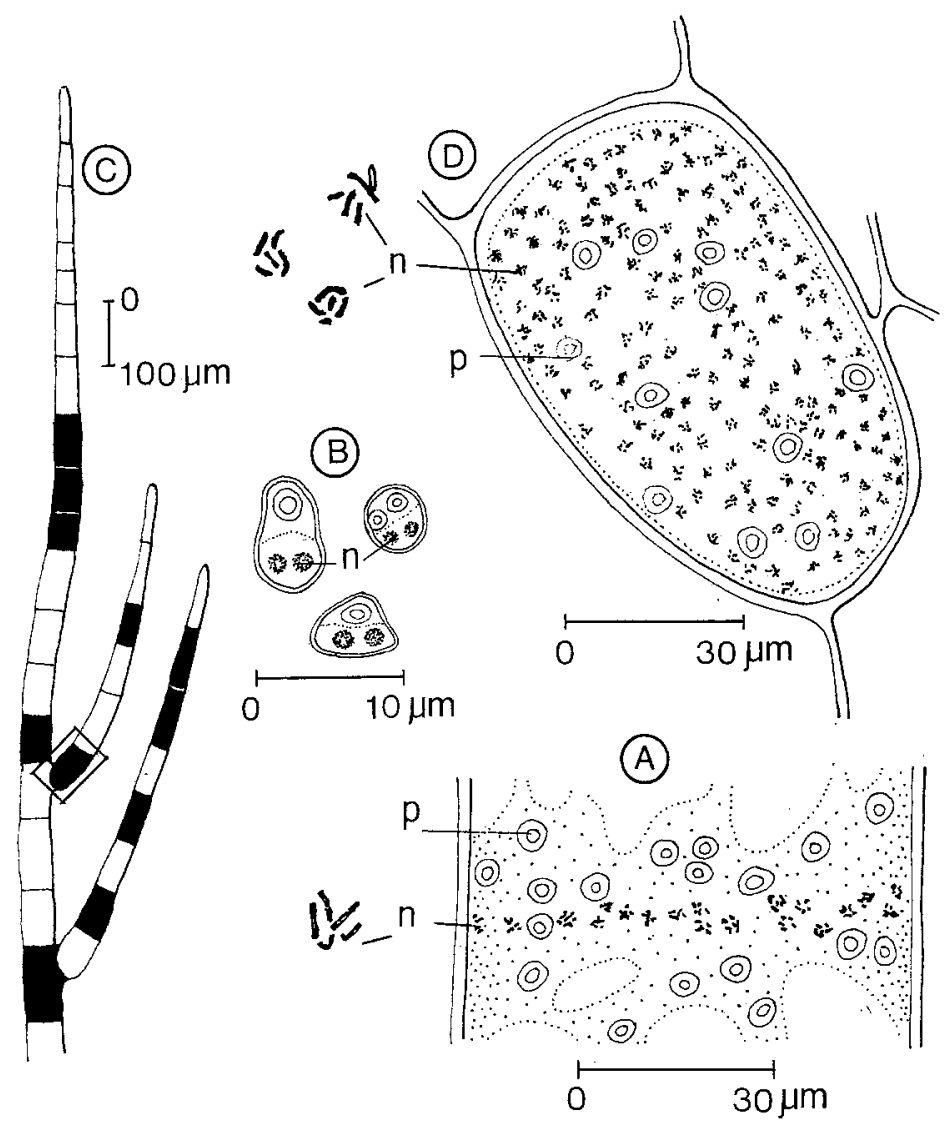

Fig. 2: Acrosiphonia arcta. Plante provenant de la nature. $A$ portion d'une cellule végétative intercalaire montrant les divisions somatiques des noyaux et l'existence de 5 chromosomes par noyau. $B$ éléments unicellulaires binuclées trouvés sur les filaments des algues fertiles et correspondant probablement aux zygotes formés dans la nature (cf. Fig. $3 \mathrm{D}$ ). C fragment de thalle devenu fertile en culture; gamétocystes en noir. $D$ détail du gamétocyste encadré en $C$; divisions simultanées des noyaux gamétogènes; 5 chromosomes par noyau. n: noyau, $p$ : pyrénoïde (FEULGEN)

D'autres rameaux fertiles de ce même matériel ont ensuite été placés en cultures unialgales. Leurs gamétocystes n'ont pas tardé à émettre abondamment des gamètes biflagellés comme dans le cas précédent. Mais contrairement à ce qui avait alors été observé, ces gamètes ont copulé très activement en formant des planozygotes à quatre flagelles et à deux stigmas (Fig. 3 A). Ces planozygotes se sont immobilisés du côté 
opposé à la lumière, occasionnellement vers la lumière. Ils ont été ensemencés sur lames, ce qui a permis l'obtention de plusieurs lots de cultures unialgales contenant chacun un grand nombre de zygotes. Ces cultures ont ensuite été placées à $+15^{\circ} \mathrm{C}$.

\section{Développement des zygotes en culture et comportement des noyaux gamétiques}

Ces observations ont été réalisées d'une part au moyen de fixations et colorations de zygotes au cours de leur développement, par la méthode précédemment employée, et d'autre part, par le maintien des zygotes en culture, à titre de témoins.

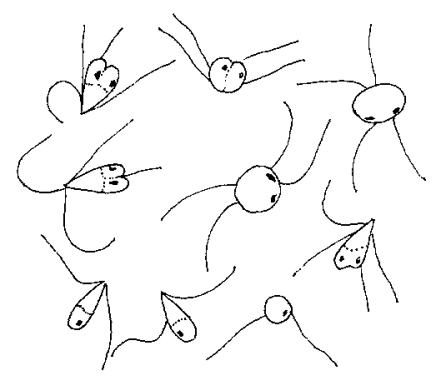

(A)
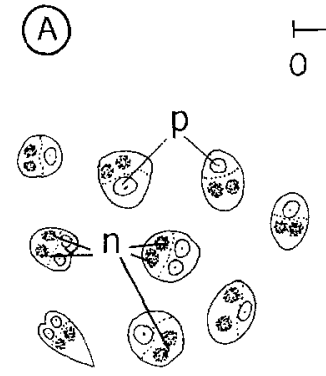

(C)

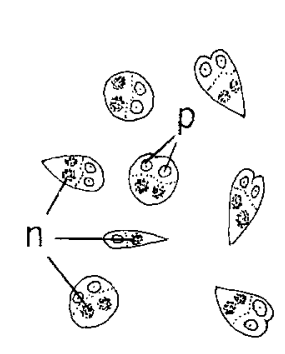

(B)

Fig. 3: Acrosiphonia arcta. A gamètes et planozygotes quadriflagellés (OsO4 $2 \%$ ). B, C, D zygotes et gamètes âgés respectivement de 24 heures, 3 jours et 8 jours, montrant deux noyaux dans chaque élément. n: noyau, p: pyrénoïde (FEULGEN)

Après 24 heures de culture, la plupart des zygotes fixés et colorés sont encore en forme de coeur (Fig. 3 B). Cependant la plasmogamie est achevée de même que la fusion des deux plastes qui forment maintenant un plaste unique renfermant les deux pyrénoïdes initiaux. Quant aux noyaux provenant de chaque gamète, colorés au rouge pourpre, ils sont parfaitement séparés l'un de l'autre. Les gamètes restants, par contre, beaucoup moins volumineux que les zygotes, apparaissent nettement uninucléés.

Quand les zygotes ont 3 jours ils offent pour la plupart un aspect arrondi (Fig. $3 \mathrm{C}$ ). On observe alors un seul pyrénoïde dans le plaste. Mais tous les zygotes renferment encore les deux noyaux qui sont totalement indépendants et quiescents. 

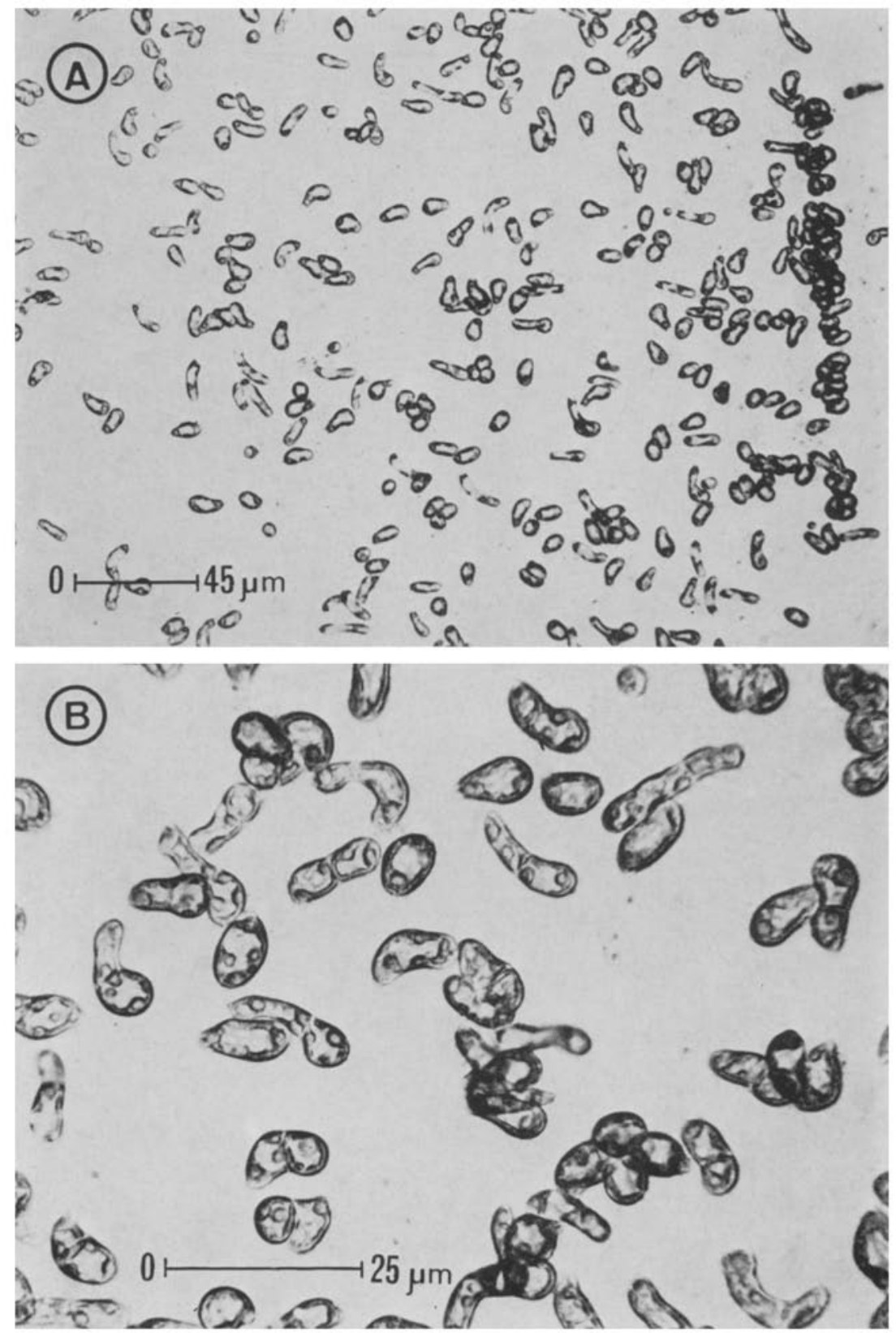

Fig. 4: Acrosiphonia arcta. Développement des faux zygotes en culture. $A$ culture âgée de 10 jours; faux zygotes à l'état siphoné; on note l'absence d'éléments codiolö̈des. $B$ culture âgée de 15 jours; les faux zygotes ont, pour la plupart, subi un cloisonnement transversal. (Observation vitale) 
Au bout de 8 jours de culture, cette situation est fondamentalement inchangée (Fig. 3 D). Les zygotes sont un peu plus gros que dans le cas précédent, mais les deux noyaux gamétiques, toujours jumelés, n’ont pas fusionné. De même ils sont toujours au repos.

La Figure 4 A montre l'aspect des zygotes à l'état vivant, après 10 jours de culture. Si l'on peut constater une légère différence en ce qui concerne la vitesse de croissance des zygotes, on peut, néanmoins, remarquer que leur mode de développe-

(A)
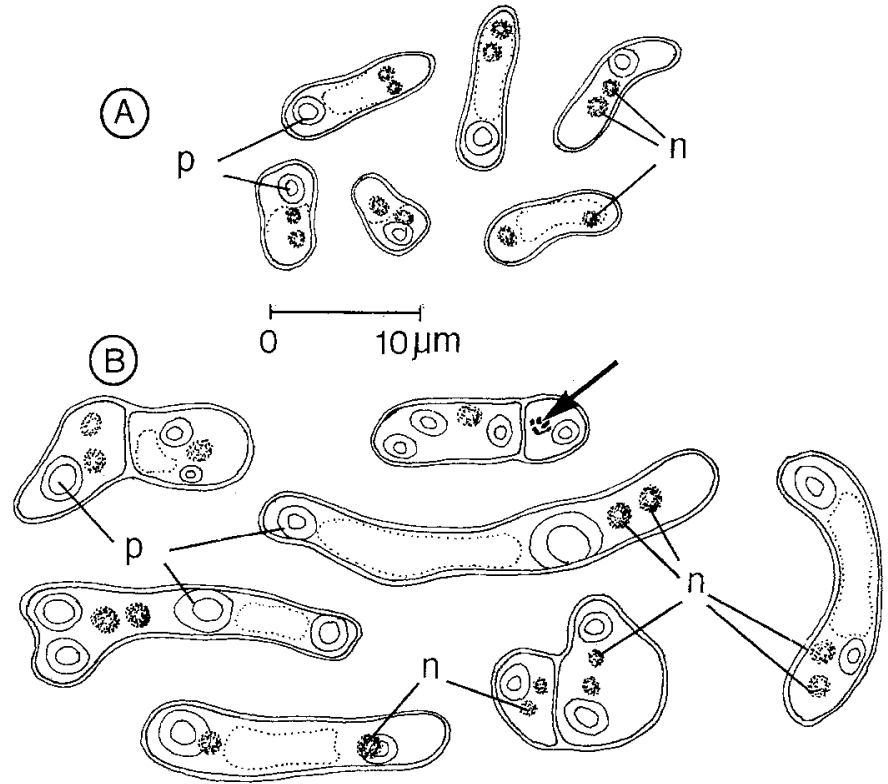

Fig. 5: Acrosiphonia arcta. A zygotes âgés de 10 jours; deux noyaux par élément. $B$ zygotes après 15 jours de culture; existence d'éléments non cloisonnés, chacun à deux noyaux, et d'éléments cloisonnés renfermant au minimum un noyau par cellule; la flèche indique environ 5 chromosomes lors d'une première activité nucléaire observée dans les germinations zygotiques. n: noyau, p: pyrénoïde (FeulgeN)

ment est analogue. Ils tendent simplement à s'allonger, ce qui s'accompagne de l'étirement $\mathrm{du}$ contenu cellulaire et la fenestration du plaste. On constate aussi l'absence totale d'éléments codioloïdes dans cette culture.

Dans un lot de zygotes de même âge (Fig. 5 A) on observe, après fixation et coloration, que chaque élément est encore nettement binucléé et qu'il n'y a aucun indice de multiplication, ni de dégénérescence au niveau des noyaux. Dans quelques cas, ceux-ci se trouvent, cependant, écartés l'un de l'autre.

$\mathrm{Au}$ bout d'une quinzaine de jours de culture, les zygotes ont formé des éléments allongés ou plus ou moins trapus, dont certains se sont déjà cloisonnés (Fig. 5 B). Dans les éléments non encore cloișonnés, de nouveaux pyrénoïdes se sont élaborés, tandis que les noyaux sont toujours au nombre de deux, soit jumelés, soit éloignés l'un de l'autre. Quant aux éléments cloisonnés, on y trouve au minimum un seul noyau dans chaque cellule. De plus, pour la première fois, on observe une activité 

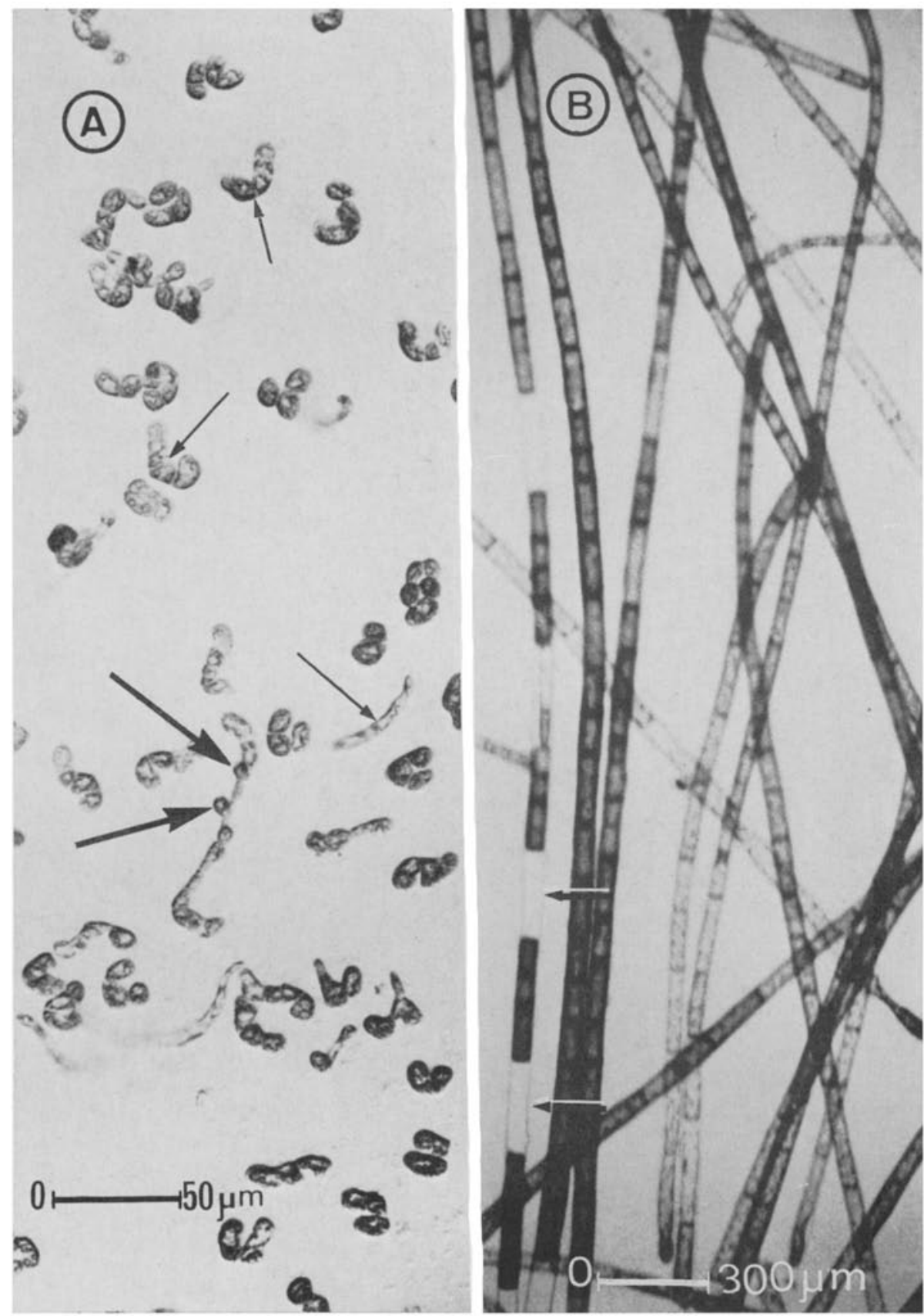

Fig. 6: Acrosiphonia arcta. Développemenc des faux zygotes en culture. A culture âgáe de 24 jours; protonémas rampants (flèches minces); ébauches de frondes dressées (flèches épaisses). $B$ culture âgée de 2 mois; plantes à l'état fertile; gamétocystes vides (flèches). (Observation vitale) 
au niveau des noyaux permettant, dans quelques cas, de déterminer le nombre chromosomique: celui-ci s'avère égal à cinq. On remarque aussi que l'une des cellules peut contenir deux noyaux alors que l'autre en renferme un seul. Ceci suggère que les divisions nucléaires ne sont pas nécessairement synchrones dans les deux cellules. La Figure $4 \mathrm{~B}$ montre la germination des zygotes à l'état vivant à ce même stade. On n'y décèle aucune trace d'éléments codioloïdes.

L'évolution des zygotes continue ensuite par la multiplication des cellules et la formation d'un appareil rampant. Celui-ci est le plus souvent filamenteux comme en témoigne la Figure $6 \mathrm{~A}$. Il forme par la suite, quelques ébauches qui se développent en frondes dressées, d'abord simples, puis ramifiées de façon tout à fait analogue à ce qui a déjà été đécrit et figuré chez cette espèce par KorNmANN $(1964,1965)$.

Les plantes ainsi obtenues présentent tous les caractères structuraux de l' $A$. arcta quoique leur morphologie soit plus anarchique que chez les plantes récoltées dans la nature. Le nombre chromosomique de leurs noyaux ne diffère pas de celui trouvé chez les plantes-mères (Fig. $7 \mathrm{~A}$ ).

Ces plantes ont fructifié en culture après traitement au froid de l'ordre de $+6^{0}$ $\grave{a}+8^{0} \mathrm{C}$ (Fig. $6 \mathrm{~B}$ ). Pendant trois générations successives elles se sont comportées, en culture, comme les plantes-mères. En aucun cas il n'a été possible de déceler dans les cultures des éléments codioloïdes, mais seulement des plantes filamenteuses.
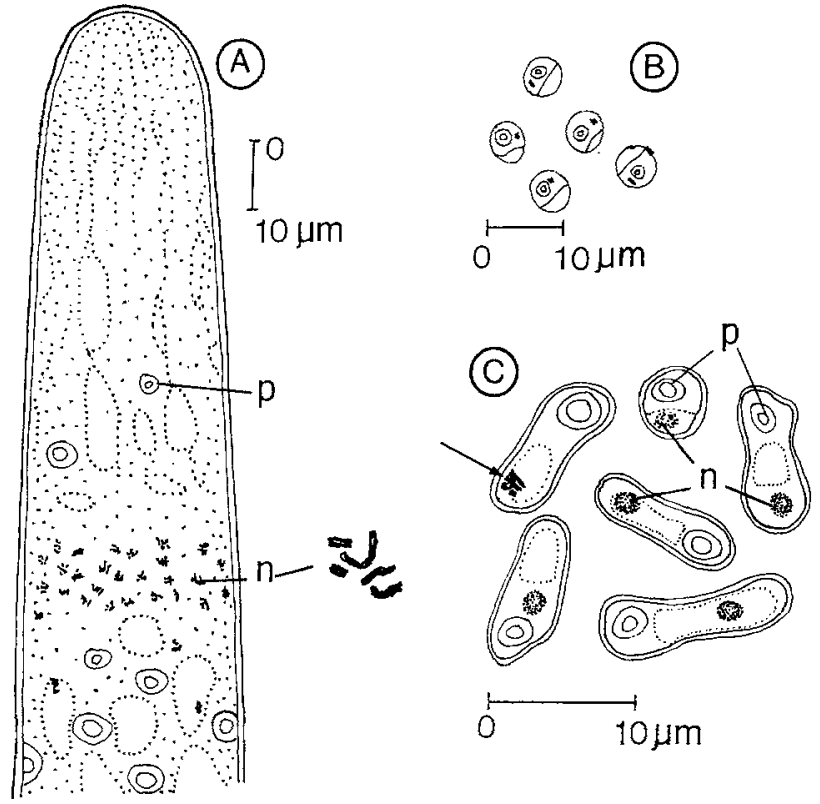

Fig. 7: Acrosipbonia arcta. A fragment d'une cellule terminale d'une plante obtenue en culture à partir d'un faux zygote; divisions somatiques des noyaux et existence de 5 chromosomes par noyau (FEULGEN). $B$ germination de gamètes obtenus dans un lot où aucune copulation n'a été observée (observation vitale). C germinations d'origine gamétique après environ 15 jours de culture; un seul noyau par cellule et la première division de celui-ci (flèche), n: noyau, p: pyrénoïde (FeUlgeN) 


\section{Evolution des gamètes en culture}

Les gamètes obtenus au début de ces études n'ont manifesté aucun signe de copulation. Il reste à expliquer ce curieux comportement qui pourrait être dû au milieu confiné dans lequel se trouvaient les algues durant le transport, à l'obscurité, au vieillissement des gamètes émis, ou à d'autres facteurs inconnus. Quoiqu'il en soit l'immense majorité de ces gamètes ont dégénéré en culture. Cependant quelques très rares éléments, issus de gamètes apparemment normaux, munis chacun d'un seul stigma et d'un pyrénoïde se sont développés directement conme les zygotes binucléés (Fig. 7 B). Mais contrairement à ces derniers, ces éléments possèdent, chacun, comme on pouvait $s^{\prime} y$ attendre, un seul noyau dont la première division apparait purement mitotique, révélant l'existence de 5 chromosomes environ (Fig. $7 \mathrm{C}$ ).

Le développement des éléments gamétiques en culture a été plus lent que celui des zygotes binucléés. Cependant les plantes obtenues ont fructifié en donnant des gamètes qui, cette fois-ci, ont copulé normalement en formant des zygotes qui ont produit des plantes filamenteuses.

\section{DISCUSSION}

Ces études montrent que les éléments résultant de la copulation des gamètes chez l'Acrosiphonia arcta sont susceptibles de se développer directement en plantes filamenteuses sans passer par le stade codiolöde et sporophytique caractérisant le cycle digénétique et hétéromorphe rencontré chez cette espèce ainsi que chez l'Acrosiphonia spinescens de Roscoff. Ces plantes correspondent ainsi à l'espèce désignée par KoRNMANN (1965) sous le nom d'Acrosiphonia arcta, forme A, et qui, selon cet auteur, aurait un cycle monogénétique et diplophasique («Diplont»). Or il est démontré ici, par des numérations chromosomiques au niveau des noyaux tant somatiques que gamétogènes, que ces plantes ne sont pas diploïdes, mais haploïdes possédant des noyaux à $\mathrm{n}=5$ chromosomes. Il faut noter que ce nombre est le même que celui établi au moment de la méiose dans les sporophytes codioloïdes de l'Acrosipbonia spinescens (Jónsson 1970). Ces plantes ne font donc pas partie d'un cycle diplontique comme l'a affirmé Kornmann (1965).

L'observation de zygotes constamment binucléés, issus de plasmogamie caractérisée, constitue un fait tout à fait remarquable chez l'A. arcta. Au cours des différents stades de développement des zygotes, on n'a, en effet, jamais observé la fusion des noyaux gamétiques, et, à aucun moment, il n'a été possible de rencontrer des zygotes à un seul noyau suggérant que la caryogamie ait eu lieu. Les zygotes peuvent être, au contraire, binucléés jusqu'au premier cloisonnement de l'élément zygotique, qui intervient au bout d'une quinzaine de jours. A ce moment les deux pronuclei se trouvent définitivement séparés, comme le témoigne l'existence d'éléments zygotiques bicellulaires dont chacune des deux cellules ne contient au minimum qu'un seul noyau. Si vraiment il y avait eu fusion incontrôlée de deux noyaux gamétiques, celle-ci aurait dû être suivie tôt ou tard de méiose, comme cela se passe, par exemple, dans 
les zygotes uninucléés du cycle monogénétique et haplophasique de l'Acrosiphonia sonderi d'Islande (JónSson 1969). Or aucun signe de méiose n'a été observé. Ces constatations plaident ainsi en faveur de la suppression totale de la caryogami dans ce cas.

En outre, il est important de remarquer que, dans le cycle digénétique et hétéromorphe de l'A. spinescens, il peut y avoir formation simultanée, à partir d'un même gamétophyte isolé, de vrais zygotes uninucléés qui, sans exception, donnent des sporophytes codioloïdes, et d'autre part, de faux zygotes binucléés qui, de façon tout à fait analogue à ce qui s'observe chez l'A. arcta, donnent directement des nouvelles plantes d'Acrosipbonia (Jónsson 1964). Dans ce cas, il est impensable qu'il y ait caryogamic dans les zygotes binucléés à développement non codiolö̈de, à moins d'admettre, ce qui n'est pas possible, l'existence de deux sortes de cycles sexués fondamentalement différents chez le même individu ainsi que deux localisations différentes de la méiose au sein d'un tel «cycle». Cette constatation rejoint celle précédemment formulée quant à la réalité apocaryogamique dez zygotes de l'A. arcta étudié ici.

Il faut noter que les deux noyaux gamétiques, désormais séparés après le premier cloisonnement du zygote, évoluent par la suite, indépendamment, par des divisions successives, d'abord dans le protonéma, ensuite dans les frondes qui s'élèvent à partir de cellules de ce protonéma. Chaque fronde dressée possède ainsi une seule catégorie de noyaux, provenant respectivement de l'un ou de l'autre des noyaux primitifs. Il en résulte que l'ensemble de l'organisme ainsi constitué est micto-haplö̈de, tandis que les cellules qui le composent sont haplö̈des. La micto-haploïdie chez ces algues diffère donc de celle observée chez certains champignons où les deux noyaux antagonistes restent jumelés dans les cellules. Il est intéressant de remarquer à ce propos que, si la micto-haploïdie se réalisait dans une algue de structure siphonée, les deux catégories de noyaux se trouveraient mélangées dans le même thalle, ce qui présenterait alors des analogies frappantes avec ce qui se passe, dans certains cas, chez les champignons.

Quant aux gamètes qui ne copulent pas, il est certain qu'ils dégénèrent en immense majorité. Cependant, les quelques éléments uninucléés trouvés dans des lots où aucune copulation n'a été observée, laissent à penser que les gamètes puissent, dans des proportions extrèmement faibles, se développer directement. Cette constatation qui est en accord avec les observations de KorNMANN (1962) doit être rapprochée de celle faite chez l'A. spinescens où l'on a signalé des éléments non codioloüdes à un seul noyau en voie de division (Jónsson 1962). Il est évident que, dans ce cas, il ne s'agit pas de vrais ou de faux zygotes.

Les plantes obtenues à partir de faux zygotes de l' $A$. arcta continuent à se reproduire en culture directement, de la même façon que les plantes-mères. Est-ce qu'il en est de même dans la nature? Ceci parait certain. En effet, selon KornmanN (1965), les plantes d'A. arcta récoltées à plusieurs reprises en différents endroits à Héligoland se reproduisent invariablement sans jamais former de sporophytes codioloïdes, ceux-ci n'ayant été obtenus qu'une seule fois à partir d'une seule plante, née en culture, de zoïde issu d'endophyte de Petrocelis. Ceci indique donc que la population naturelle de I'A. arcta d'Héligoland est essentiellement composée de lignées micto-haploïdes stables et distinctes. La présence d'éléments binucléés trouvés sur les plantes-mères et analogues aux faux zygotes d'une dizaine de jours plaide aussi en faveur de cette constatation. 
Ces résultats posent des problèmes intéressants non résolus. On peut ainsi se demander quelle est l'origine des lignées micto-haploïdes de l' $A$, arcta d'Héligoland. A priori on pourrait penser que ces plantes sont initialement issues du cycle sexué hétéromorphe qui s'observe dans cette région par un mécanisme régressif de la sexualité. Or, il n'a pas encore été démontré que des plantes micto-haploïdes stables dérivent actuellement de ce cycle. Kornmann (1965) a bien obtenu à partir de l'unique gamétophyte connu à Héligoland quelques rares plantes filamenteuses d'origine mal déterminée. Mais ces plantes ne semblent pas conduire à la formation de lignées stables car elles produisent, au cours des générations ultérieures, des sporophytes codioloïdes (communication personnelle de Kornmann). Ceci rejoint d'ailleurs la constatation faite sur la population de l'A. spinescens des côtes françaises, où les plantes micto-haploïdes, nées dans le cycle hétéromorphe, effectuent un retour au cycle sexué normal, dès la première génération. Il en résulte que, si le cycle hétéromorphe peut ainsi être le siège de ségrégation de plantes micto-haploïdes, on ne voit pas, dans l'état actuel des choses, comment ces plantes peuvent s'isoler pour former des lignées distinctes analogues à celles rencontrées chez l'A. arcta.

Un autre problème est celui relatif à la présence presque exclusive de plantes micto-haploïdes dans la population de l'A. arcta d'Héligoland, alors que sur les côtes françaises de telles lignées n'ont pas été trouvées. Est-ce que cette différence a une signification phylogénétique? Cela ne parait pas invraisemblable. Il est en effet possible que nous soyions en présence de deux étapes de l'évolution régressive du cycle sexué fondamental. La première étape est celle observée à Roscoff où le cycle sexué initial est fondamentalement conservé. Il n'est perturbé que facultativement par une reproduction accessoire micto-haploïde qui d'ailleurs ne paraît pas laisser trace dans la nature, étant donné la possibilité de normalisation des plantes micto-haploïdes. A Héligoland, au contraire, l'évolution régressive par apocaryogamie aurait franchi un pas décisif: l' espèce y a pratiquement perdu l'aptitude à former des sporophytes codioloïdes, ce qui se traduit par l'extrême raréfaction du cycle sexué fondamental dans la nature. La dégradation sexuelle aurait ainsi été compensée dans cette région par un développement micto-haploïde prépondérant et stable. La tendance évolutive du cycle primitif chez ces algues se présente donc comme un rapport de force entre le cycle sexué fondamental et le cycle parasexué micto-haploïde.

On peut enfin se poser la question relative à la cause profonde de la défaillance caryogamique qui est à la base de l'évolution micto-haplö̈de observée chez ces algues.

A ce propos il faut souligner que le phénomène de l'apocaryogamie n'est pas en rapport avec quelques fluctuations du milieu extérieur, étant donné que le même individu est susceptible de donner simultanément des faux et des vrais zygotes. La possibilité d'anomalies structurales, telle que la séparation incomplète des gamètes, doit être écartée. Ceci est particulièrement évident chez l'A. arcta qui est capable de former exclusivement et en grande abondance des faux zygotes, après copulation normale des gamètes. Par conséquent, il est permis de supposer que le blocage de la caryogamie et le processus de la ségrégation apocaryogamique soient sous la dépendance de facteurs intrinsèques, peut-ètre d'ordre mutationnel, opérant initialement, soit au moment de la différenciation des gamètes, soit au moment de la méiose. La normalisation de la reproduction chez les plantes micto-haploïdes de l'A. spinescens 
permettrait, en outre, d'envisager la possibilité de réversion spontanée de ce phénomène.

Cette hypothèse peut rendre compte des faits rapportés. Mais il parait prématuré, dans l'état actuel des observations, et notamment en l'absence d'analyse génétique, de formuler une interprétation précise quant au mécanisme intime responsable de la ségrégation apocaryogamique.

\section{RESUME}

1. La caryogamie est supprimée dans des zygotes obtenus à partir d'une souche sauvage unialgale de l'Acrosiphonia arcta (Dillw.) J. Ag. de Héligoland.

2. Les deux pronucléi des pseudozygotes ainsi formés peuvent se séparer définitivement au moment de la première segmentation de l'élément zygotique qui intervient au bout d'une quinzaine de jours.

3. Les pseudozygotes se développent directement en nouvelles plantes d'Acrosipbonia.

4. Les plantes issues de pseudozygotes ainsi que les plantes-mères possèdent des noyaux haploïdes avec $\mathrm{n}=5$ chromosomes.

5. L'Acrosiphonia arcta d'origine apocaryogamique semble constituer des lignées micto-haploïdes stables et génétiquement isolées. Leur origine et leur mode de ségrégation sont discutés.

\section{LIT'TERATURE CITEE}

Jónsson, S., 1959. L'existence de l'alternance hétéromorphe de générations entre l'Acrosiphonia spinescens $\mathrm{K}_{\mathrm{JELl}}$. et le Codiolum petrocelidis Kück. C. $\mathrm{r}$. hebd. Séanc. Acad. Sci., Paris 248, 835-837.

- 1962. Recherches sur des Cladophoracées marines (structure, reproduction, cycles comparés, conséquences systématiques). Annls Sci. nat. (Botanique) 3, 25-230.

- 1964. Existence d'une caryogamie facultative dez l'Acrosiphonia spinescens (Kürz.) KJellm. C. r. hebd. Séanc. Acad. Sci., Paris 258, 6207-6209.

- 1968. Sur l'existence de lignées micto-haplö̈des dans la population naturelle de l'Acrosiphonia arcia (Dıllw.) J. Ag. d' Héligoland. C. r. hebd. Séanc. Acad. Sci., Paris (D) 267, $53-55$.

- 1969. Le cycle évolutif de l'Acrosiphonia sonderi (Kütz.) Kornm. d'Islande et l'origine de ses races asexuées. Revue gén. Bot. 76, 267-286.

- 1970. Localisation de la méiose dans le cycle de l'Acrosiphonia spinescens (KüTz.) KJELlu. (Acrosiphoniacées). C. r. hebd. Sćanc. Acad. Sci., Paris (D) 271, 1859-1861.

Kornmann, P., 1962. Eine Revision der Gattung Acrosiphonia. Helgoländer wiss. Meeresunters. 8, 219-242.

- 1964. Der Lebenszyklus von Acrosiphonia arcta. Helgoländer wiss. Meeresunters. 11, 110-117.

- 1965. Was ist Acrosiphonia arcta? Helgoländer wiss. Meeresunters. 12, 40-51.

- 1970. Phylogenetische Beziehungen in der Grünalgengattung Acrosipbonia. Helgoländer wiss. Meeresunters. 21, 292-304.

Adresse de l'auteur: Dr. S. Jónsson

Laboratoire de Biologie Végétale Marine

Faculté des Sciences

7, quai Saint-Bernard

Paris 5 e, France 